\title{
Performance of multicarrier complementary- coded CDMA under frequency-selective Nakagami-m fading channels
}

\author{
D. Judson ${ }^{1 *}$ and A. Albert Raj ${ }^{2}$
}

\begin{abstract}
Multicarrier complementary-coded code division Multiple Access (MC CC-CDMA) is becoming an attractive multiple access technique for high data rate transmission in future wireless communication systems. MC CC-CDMA systems transmitting over frequency-selective channels suffer from multiple access interference (MAI) owing to non-ideal correlation properties of complementary codes (CC). MC CC-CDMA with equalization has recently gained much attention for its ability to offer an excellent performance than traditional systems in frequency-selective fading channels. In this paper, the authors present an analytical study and investigation of MC CC-CDMA downlink system using different combining schemes. The use of parallel interference cancellation (PIC) under frequency-selective Nakagami-m fading channels is also analyzed. A comparison among different combining schemes is provided to show the impact of PIC with minimum mean square error combining (MMSEC) and maximal ratio combining (MRC) on the performance of MC CC-CDMA system. The analytical and simulation results show that the combination of general combining schemes with PIC provides an efficient solution to suppress MAI in downlink MC CC-CDMA system than conventional MC-CDMA systems using Walsh codes under frequency-selective channels.
\end{abstract}

Keywords: Code division multiple access (CDMA), Complementary codes (CC), Multiple access interference, Frequency-selective fading, Nakagami-m fading

\section{Introduction}

Next generation wireless communication is expected to provide multimedia services with different quality of service, offering high throughput and reliability under limited power and bandwidth resources. The main impairments limiting the capacity and maximum data rate offered to the end users of wireless communication system are multipath fading, delay spread, and multiple access interference (MAI). Multiple access (MA) techniques allow different users to share the same channel effectively as possible. Direct sequence code division multiple access (DS-CDMA) is one of the MA techniques for its improved performance in terms of system

\footnotetext{
* Correspondence: judson_d2001@yahoo.co.in

${ }^{1}$ Department of Electronics and Communication Engineering, St. Xavier's Catholic College of Engineering, Chunkankadai 629003Tamilnadu, India Full list of author information is available at the end of the article
}

capacity and coverage compared to time division multiple access and frequency-division multiple access [1].

In CDMA, each user uses a unique code sequence that allows multiple users to share the system bandwidth. The codes used for CDMA should have low or zero cross-correlation in order to overcome multiple access interference and multipath interference, which limits the capacity of the current 3G system to half of its processing gain (PG) [2]. The major limitation of existing signature sequences [3], such as Gold codes, M-sequence, Kasami sequence, and Walsh-Hadamard sequence, are that they are no longer orthogonal in real-time scenarios such as uplink transmission, multiple access, and multipath interference which reduces the system capacity, initiating the need for perfect orthogonal codes.

In [4], orthogonal complementary codes (OCC) were introduced which offer zero autocorrelation (except zero shift) and zero cross-correlation for all possible shifts

\section{实 Springer}

(c) 2016 Judson and Raj. Open Access This article is distributed under the terms of the Creative Commons Attribution 4.0 International License (http://creativecommons.org/licenses/by/4.0/), which permits unrestricted use, distribution, and reproduction in any medium, provided you give appropriate credit to the original author(s) and the source, provide a link to the Creative Commons license, and indicate if changes were made. 
between any pair of OCC. Here, the orthogonality is preserved by assigning a flock of element codes to a user instead of a single code as in traditional CDMA systems with unitary codes. The use of complete complementary codes for CDMA was investigated using quadrature amplitude modulation in [5]. Most of the attractive features of OCC were reported in $[6,7]$ to show the superiority of complementary codes in multipath and multiuser scenarios. In [8], a superset of perfect complementary codes was proposed to increase the set size of the OCC to its processing gain. In order to achieve interference-free OCC, the flock size of the signature code must be equal to the set size of the codes. This problem was addressed in [9] where the code generated was able to support more users compared to complete complementary codes.

In a complementary-coded (CC) CDMA, each user data is spread with $N$ element codes of a particular complementary code and transmitted using $N$ independent subcarriers [10] to form a multicarrier CC-CDMA system. At the receiver, the signals received using different subcarriers are passed to their corresponding chip matched-filters and summed up with an equal gain to reconstruct ideal correlation properties. Such equal gain summation is hard to achieve over frequency-selective fading channels, where different subcarriers may suffer from different fading levels.

The ideal orthogonality of complementary correlation function under frequency-selective fading channels is lost due to multiple access interference and multipath interference existing between users transmitting over a common bandwidth. The performance degradation caused due to channel selectivity in MC CC-CDMA was addressed for both downlink [11] and uplink [12] under Rayleigh fading channels. Even though channel selectivity reduces orthogonality of $\mathrm{CC}$, the multi-sequence spreading and summation at the receiver of MC CC-CDMA system offers additional diversity gain which cannot be achieved in traditional CDMA systems using single carrier.

The combining algorithms play a vital role in enhancing the performance of MC CC-CDMA systems. Equal gain combining (EGC) works well only in correlated channel conditions. In frequency-selective fading conditions, orthogonal restoring combining (ORC), maximal ratio combining (MRC), and minimum mean square error combining (MMSEC) have been a promising technique in reducing MAI [13]. In [14], multiple-input and multiple-output orthogonal frequency-division multiplexing systems were proposed for downlink transmission using orthogonal variable spreading factor codes to obtain spatial diversity for existing single-input and single-output systems. Different types of gain combining schemes were employed in frequency domain to recover the data symbols of desired code channels.
In our study, we aim to enhance the performance of downlink MC CC-CDMA system by mitigating MAI in frequency-selective Nakagami-m fading channels. The impacts of different combining schemes such as ORC, MRC, and MMSEC on bit error rate (BER) performance of CC-CDMA are examined under frequency-selective fading channels. Parallel interference cancellation (PIC) with MRC and MMSEC is suggested for obtaining MAIfree performance in CC-CDMA systems with uncorrelated channel gain in each subcarrier under frequencyselective Nakagami-m fading channels. The accuracy of the derived BER expressions is verified with the simulated ones for MC CC-CDMA systems with different combining methods under different system parameters. Further, we compare the BER performance of complementarycoded CDMA with conventional CDMA systems employing Walsh codes to show the advantage of CC-CDMA in obtaining large diversity gain when incorporating PIC with different combining schemes.

The rest of the paper is organized as follows: in Section 2, we describe the system model of MC CCCDMA system under frequency-selective Nakagami-m fading channels. Section 3 provides finite summation expressions and the resultant performance analysis of CC-CDMA using different combining schemes. The analytical and simulation results with discussions are presented in Section 4. Finally, Section 5 presents the conclusions.

\section{MC CC-CDMA system model}

In this section, the system model for MC CC-CDMA is discussed.

\subsection{CC-CDMA transmitter descriptions}

The transmitter section for MC CC-CDMA is shown in Fig. 1 . There are $K$ users in the multiple access system. The $k$ th user information symbol after baseband modulation denoted as $a_{k}(t)$ is a sequence of positive and negative pulses of duration $T_{b}$ with amplitude +1 or -1 . The baseband modulated symbols of user $k$ are spread with a flock of element codes represented as

$$
C^{(k)}=\left[C_{1}^{(k)}, \quad C_{2}^{(k)}, \ldots C_{N}^{(k)}\right]^{T}
$$

where $k=1,2, \ldots, K$, and $n=1,2, \ldots, N$.

Here, $C^{(k)}$ and $C_{n}^{(k)}$ represent the complementary code and the corresponding element code, respectively. $K$ and the flock size $N$ denote the number of users and number of element codes used by the same user, respectively.

In a conventional synchronous CDMA system, each user uses a unique orthogonal spreading code such as the Walsh code to spread its information symbols and 


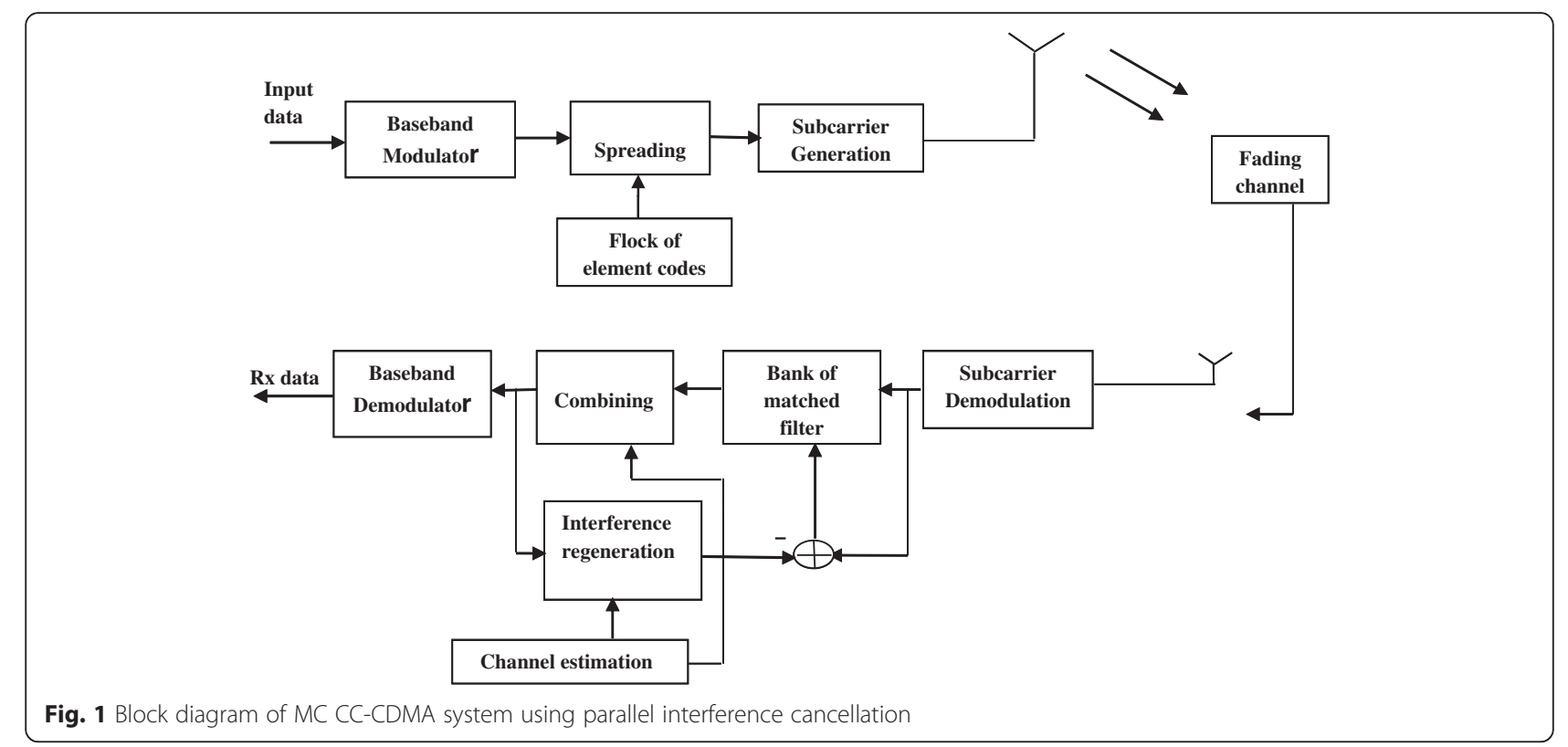

its processing gain is equal to the length of the unitary code. Walsh codes are commonly used in downlink channels due to better cross-correlation property of code set. Synchronous users modulated using Walsh codes can be separated at the receiver with less interference as long as its orthogonality is not corrupted due to MAI. However, in CDMA system with CC, the orthogonality of each complementary code $C^{(k)}$ is based on a flock of element codes offering zero cross-correlation characteristics than conventional CDMA system. The processing gain of a CDMA system with $\mathrm{CC}$ is the product of flock size and the length of element code.

In MC CC-CDMA, the spreading data matrix of user $k$ is given by

$$
S(k)=\left[\begin{array}{cccc}
a_{k 1} C_{1}{ }^{(k)} & a_{k 2} C_{1}{ }^{(k)} & \cdots & a_{k N} C_{1}{ }^{(k)} \\
a_{k 1} C_{2}{ }^{(k)} & a_{k 2} C_{2}\left({ }^{(k)}\right. & \cdots & a_{k N} C_{2}{ }^{(k)} \\
a_{k 1} C_{3}{ }^{(k)} & a_{k 2} C_{3}(k) & \cdots & a_{k N} C_{3}{ }^{(k)} \\
\vdots & \cdots & \vdots \\
\vdots & \cdots & \vdots \\
a_{k 1} C_{N}{ }^{(k)} & a_{k 2} C_{N}{ }^{(k)} & \cdots & a_{k N} C_{N}{ }^{(k)}
\end{array}\right]
$$

where $\left[a_{k 1}, a_{k 2} \ldots, a_{k N}\right]$ represents the data corresponding to user $k$.

The spreaded data of user $k$ is sent through multiple carriers to the receiver. The transmission using multiple carriers provides ideal autocorrelation function and cross-correlation function behaviors. In spite of the occurrence of many side lobes during autocorrelation among individual element codes, the sum of correlation between $N$ element sequences of two different complementary codes is zero, as given below

$$
\sum_{n=1}^{N} \rho\left(C_{n}^{(k)} C_{n}^{(l)} ; \tau\right)=\left\{\begin{array}{l}
N L_{c}, \tau=0 ; k=l \\
0, \tau \neq 0
\end{array},\right.
$$

where $0 \leq \tau<L_{c}$ represents the relative chip delay between the local correlator and the received signal. $L_{c}$ represents the length of the element code, and $N$ denotes the number of element codes. The data $a_{k}(t)$ spread with $N$ element codes followed by subcarrier modulation is denoted as

$$
S^{(k)}(t)=\sqrt{p} \sum_{n=1}^{N} a_{k}(t) C_{n}^{(k)}(t) e^{j \varphi_{n}}
$$

where $p=\frac{E_{b}}{N T_{b}}$ and $T_{b}$ represent the transmitter power and the bit interval given by $T_{b}=L_{c} T_{c}$, respectively. $E_{b}$ and $T_{c}$ are the bit energy and chip interval, respectively. $e^{j \varphi_{n}}$ is the subcarrier assigned to $n$th element code. $C_{n}^{(k)}$ $(t)$ is the spreading waveform of user $k$ defined as

$$
C_{n}^{(k)}(t)=\sum_{m=0}^{L c-1} C_{n, m}^{(k)} \Phi\left(t-m T_{c}\right), \quad 0<t \leq T_{b},
$$

where $C_{n, m}^{(k)}$ represents the chip values of the spreading sequence assigned to user $k$. $\Phi(t)$ represents the rectangular shape of chip limited waveform.

The signal from $K$ users undergoes subcarrier modulation and transmitted synchronously over frequencyselective fading channel. Downlink transmission is considered where coherence bandwidth of each subcarrier is assumed to be smaller than the system bandwidth. Each 
subcarrier is modeled as independent flat fading channel with the bandwidth of each subcarrier being less than the coherence bandwidth. Therefore, each subcarrier does not have selectivity, and it is also assumed that the channel coefficient is constant throughout the transmission period of 1-bit duration.

\subsection{Receiver section of MC CC-CDMA}

Suppose that the desired user of interest is $l$, the demodulated signal from subcarrier $n$ in a mobile station can be written as

$$
r_{n}^{(l)}(t)=h_{n}^{(l)} \sum_{k=1}^{K} s_{n}^{(k)}(t)+n_{n}(t)
$$

where $h_{n}^{(l)}=\left|h_{n}^{(l)}\right| e^{j \varphi_{n}^{(l)}}$ represents the channel coefficient of subcarrier $n$ from the base station to the user $l . s_{n}^{(k)}(t)$ denotes the spreaded data of $n$th element code of user $k$. $n_{n}(t)$ is the zero mean white Gaussian noise with variance $N_{o} / T_{c}$ in the subcarrier $n$ and $N_{o}$ is the power spectral density of complex additive white Gaussian noise (AWGN). Here, it is assumed that the $N$ noise samples are uncorrelated.

In multicarrier CC-CDMA system, the carrierdemodulated signals of each user are passed through $N$ chip-matched filters corresponding to $N$ element sequences. If $y_{n}^{(l)}(t)$ represents the chip-matched filter output for the element sequence $c_{n}^{(l)}$ at the receiver of desired user $l$, the matched filter output for $n$th element sequence under perfect carrier, code, and bit synchronization is denoted as

$$
y_{n}^{(l)}(t)=\int_{0}^{T} r_{n}^{(l)}(t) c_{n}^{(l)}(t) d t .
$$

Substituting (4) and (5), in (6), we have

$$
y_{n}^{(l)}(t)=h_{n}^{(l)} \sqrt{p} \sum_{k=1}^{K} b_{k}(t) \rho_{k, l, 0}^{(n)}+I_{n},
$$

where $I_{n}$ represents the sampled additive white Gaussian noise with zero mean and variance $L_{c} \sigma_{n}^{2}$ expressed as

$$
I_{n}=\int_{0}^{T} n_{n}(t) c_{n}^{(l)}(t) d t .
$$

The outputs of $N$ chip-matched filters of desired user $l$ are combined before taking decision. Here, the signals received through $N$ subcarriers results in $N$-branch diversity with each branch representing replicas of source data spread by $n$th element sequence. Hence, in order to achieve ideal correlation characteristics of the received signal under frequency-selective fading channel, a suitable combining method should be employed to achieve frequency diversity gain, from the signal received over $N$ subcarriers.

\section{Interference cancellation with different receivers for MC CC-CDMA}

The discrete-time signal component at the combiner output for desired user $l$ in the $i$ th symbol interval is denoted as

$$
y_{c}^{(l)}(i)=\sum_{n=1}^{N} y_{n}^{(l)}(i) w_{n}^{(l)},
$$

where $w_{n}^{(l)}$ is the equivalent combining weight of the $n$th subcarrier for desired user $l$. $y_{n}^{(l)}(i)$ represents the discrete-time equivalent of (8).

The different types of combining techniques normally used are orthogonal restoring combining (ORC), maximal ratio combining (MRC), and minimum mean square error combining (MMSEC). ORC performs well when the number of active users is high and the system is interference limited. However, ORC will enhance noise due to the requirements of larger weights at low signal to noise ratio (SNR). The equivalent weight for ORC is given by

$$
w_{n}^{(l)}=\frac{1}{h_{n}^{(l)}} .
$$

Using MRC, multiple uncorrelated signals at the output of $N$ matched filters are combined to obtain a frequency diversity gain. The equivalent weight corresponding to MRC is given by

$$
w_{n}^{(l)}=\left[h_{n}^{(l)}\right]^{*},
$$

where * denotes the complex conjugate operation. MMSEC is obtained based on the information of the number of active users, the noise power, and the channel gain of each subcarrier. The equivalent weight of MMSE combining is given by [15],

$$
w_{n}^{(l)}=\frac{\left[h_{n}^{(l)}\right]^{*}}{\left|h_{n}^{(l)}\right|^{2}+\frac{N N_{o}}{K_{u} E_{b}}},
$$

where $K_{u}$ is the number of active users and $E_{b} / N_{o}$ denotes the bit energy to noise density ratio.

\subsection{MRC-based parallel interference cancellation}

Multiuser detection has been an attractive technique to cancel MAI by using the information of interfering users [16] in traditional CDMA systems using unitary codes. In MC CC-CDMA, multiuser detection was not applied owing to ideal correlation characteristics of $\mathrm{CC}$, but its 
performance gets degraded in frequency-selective fading channels due to MAI existing between interfering users. Parallel interference cancellation (PIC) can be used to achieve performance improvement in multiuser CDMA systems affected due to MAI. Further, PIC using suitable interference estimation techniques such as MMSE, $\mathrm{ORC}$, and MRC can be used to estimate, regenerate, and cancel the effect of interfering users [17].

In MC CC-CDMA, PIC is applied to $N$ subcarriers to obtain per-carrier parallel interference cancellation (PCPIC). The main aims of PIC are to regenerate the signal mixed with MAI and recover the useful signal from the desired user. It is normally operated in two stages. In the first stage, the initial data estimates due to interfering users is estimated using (15) with suitable combining method and PIC is applied using (14) to regenerate and cancel MAI existing in the received signal. In the second stage, the interference-cancelled signal is combined using MRC to form PIC-MRC.

The interference-cancelled signal for subcarrier $n$, based on the estimated interference, channel gain, and the number of interfering users can be written as

$$
r_{\mathrm{pic}, n}(t)=r_{n}(t)-h_{n} \sqrt{p} \sum_{k=1, k \neq l}^{K} J^{\wedge}(k)(i) c_{n}^{(k)}(t),
$$

where $r_{n}(t), n \in\{1,2 \ldots N\}$, is the received signal for subcarrier $n$ of all the $K$ users in the system. $\hat{J}^{(k)}(i)$ represents the interference at the output of chip-matched filter from interfering user $k$ which is estimated based on specific combining weight denoted as

$$
J^{\wedge}(k)(i)=\sum_{n=1}^{N} w_{n}^{(l)} y_{n}^{(k)}(i), \quad k \neq l .
$$

After interference cancellation, the signals from $N$ subcarrier are despread using complementary code of desired user $l$ and combined to give the estimated data for element sequence $n$ as

$$
\hat{b}_{\mathrm{pic}}^{(l)}(i)=\sum_{n=1}^{N} w_{n}^{(l)} \int_{0}^{T_{b}} r_{\mathrm{pic}, n}\left(t+i T_{b}\right) c_{n}^{(l)}(t) d t
$$

Using (12) in (16), the data signal of desired user $l$ after interference estimation using a specific estimation scheme and MRC is given by

$$
\hat{b}_{\mathrm{pic}}^{(l)}(i)=L_{c} \sqrt{p} \sum_{n=1}^{N}\left|h_{n}^{(l)}\right|^{2} b^{(l)}(i)+J_{\mathrm{pic}}+N_{\mathrm{pic}}
$$

where $N_{\text {pic }}$ is the noise term and $J_{\text {pic }}$ is the residual MAI represented as

$$
J_{\mathrm{pic}}=\sqrt{p} \sum_{k=1, k \neq l}^{K}\left(b^{(k)}-J^{(k)}\right) \sum_{n=1}^{N}\left|h_{n}^{(l)}\right|^{2} \rho_{k, l, 0}^{(n)} .
$$

It can be observed from (18) that the performance of MC CC-CDMA depends on the equivalent combining weight and the correlation properties of each element sequence. In addition, the accuracy of the estimated MAI $\jmath^{(k)}(i)$ depends heavily on perfect available channel state information at the receiver of the desired user.

\subsection{Error probability of MC CC-CDMA system under Nakagami-m fading channel with MRC}

We consider downlink MC CC-CDMA communication system under frequency-selective fading channel where the signal from the base station (BS) is transmitted to $K$ simultaneous active mobile users. Each user employs $N$ element codes, and binary phase-shift keying (BPSK) is used. The flock size $N$ determines the bandwidth of each subcarrier for the fixed system bandwidth, and each subcarrier is assumed to experience slowly varying Nakagami-m fading channel. All the element codes are assumed to experience flat, but uncorrelated fading.

The error probability for MC CC-CDMA under Nakagami-m fading channel is obtained by averaging the conditional error probability with respect to $\gamma$ as follows

$$
P_{e}^{\mathrm{Naka}}=\int_{0}^{\infty} P_{e}^{\mathrm{AWGN}}(\gamma) \cdot f_{\gamma}(\gamma) d \gamma .
$$

For a Nakagami-m fading channel with parameter $\mathrm{m}$, the PDF of the fading channel with Nakagami random variable $r$ and instantaneous SNR $\gamma=r^{2} \frac{E_{b}}{N_{o}}$ over $L$ paths with MRC diversity is given by [18] as

$$
f_{\gamma}(\gamma)=\left(\frac{m}{\bar{\gamma}}\right)^{L m} \frac{\gamma^{L m-1}}{\Gamma(\mathrm{Lm})} \exp \left(\frac{-m \gamma}{\bar{\gamma}}\right) \forall \gamma>0,
$$

where $\bar{\gamma}^{\rrbracket}=E\left(R^{2}\right)$, and the parameter $m$ is defined as the ratio of moments called the fading figure [19], given by $m=\frac{\bar{\gamma}^{\mathbb{X}^{2}}}{E\left[\left(R^{2}-\bar{\gamma}\right)^{2}\right]}$.

Using (17) and (20) in (19), we get the error probability for $N$ independent subcarriers with Nakagami-m fading as

$$
\begin{aligned}
P_{e}^{\text {nak }} & =L(d)\left[\int_{0}^{\infty} Q\left(\sqrt{\frac{2 E_{b}}{N N_{o}} \sum_{n=1}^{N}\left|h_{n}\right|^{2}}\right) \gamma^{N m-1} \exp \left(\frac{-m \gamma}{\bar{\gamma}}\right) d \gamma\right], \\
& =L(d)\left[\int_{0}^{\infty}(1-\operatorname{erf}(\sqrt{\gamma})) \gamma^{N m-1} \exp \left(\frac{-m \gamma}{\bar{\gamma}}\right) d \gamma\right],
\end{aligned}
$$

where $L(d)=\frac{m^{N m}}{\Gamma(\mathrm{Nm}) \bar{\gamma}^{\mathbb{N} m}}, \gamma=\frac{2 E_{b}}{N N_{o}} \sum_{n=1}^{N}\left|h_{n}\right|^{2}$, and $L$ is replaced by $N$ for convenience. Here, erf(.) is the error 
function in probability theory obtained from [19] and is related to the Gaussian probability integral as $Q(x)=\frac{1}{2}\left(1-\operatorname{erf}\left(\frac{x}{\sqrt{2}}\right)\right)$.

Using equation (6.286/1) from [20], we get

$$
\int_{0}^{\infty}[1-\varphi(\beta x)] e^{\mu^{2} x^{2}} x^{\nu-1} d x=\frac{\Gamma\left(\frac{v+1}{2}\right)}{\sqrt{\pi} v \beta^{\nu}} F\left(\frac{v}{2}, \frac{v+1}{2} ; \frac{v}{2}+1 ; \frac{\mu^{2}}{\beta^{2}}\right),
$$

where $\beta, \mu$, and $v$ represent the constants associated with the variable $x$ in (22).

Comparing (22) with (21) and using equation (3.381/4) of [20] in (21), the error probability for MC CC-CDMA system under Nakagami-m fading channel is given by

$$
\begin{aligned}
P_{e}^{\mathrm{Nak}}= & \frac{L(d)}{2 \sqrt{\pi} N m} \Gamma\left(N m+\frac{1}{2}\right) \\
& \times F\left(N m, N m+\frac{1}{2} ; N m+1 ;-\frac{m}{\bar{\gamma}}\right)
\end{aligned}
$$

where $F(x, y ; z ; \rho)$ is the Gauss hypergeometric function [20]. The above equation can be numerically evaluated using MATLAB for each value of diversity order $N$, fad-

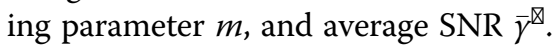

\section{Analytical and simulation results}

In this section, analytical and simulation results are presented for illustrating the performance of MC CCCDMA system under different channel conditions. In simulations, downlink communications with an uncoded BPSK modulation is considered. Here, each subcarrier is modeled as independent Nakagami-m flat fading channel and $N$ channel coefficients are considered as uncorrelated zero-mean complex Gaussian random variables with unit variance. The channel coefficients are constant throughout the transmission period of 1-bit duration. Throughout the simulation, it is assumed that perfect channel state information (CSI) is available at the receiver. We assume supercomplementary codes (SCC) in simulations, where in SCC, the flock size can be equal to their processing gain [9], thus supporting more users compared to complete complementary codes.

\subsection{A. BER performance of MC CC-CDMA without PIC}

We first illustrate the performance of MC CC-CDMA system without PIC under different fading conditions using various combining schemes such as ORC, MRC, and MMSE. The propagation channel considered is frequency-selective Nakagami-m fading channel with each subcarrier experiencing uncorrelated fading. The comparison is made using SCC having PG of 64 with flock size 16 and element code length 4 .
Figure 2 shows the BER performance of $\mathrm{MC} \mathrm{CC}$ CDMA using MRC with variations in number of users for fading parameter $m=4$ under frequency-selective Nakagami-m fading channel. It can be observed that with variations in number of users accessing the system, the effect of multiple access interference and hence BER increases. From Fig. 2, it can be seen that for BER of $10^{-2}$, the additional required SNR is $4 \mathrm{~dB}$ for 16 user system compared to eight user system. This occurs due to the increase in MAI with increase in number of users in the system and lack of suitable equalization schemes to improve the error rate performance.

Figure 3 shows the reduction in error probability with increase in fading parameter $m$ for MC CC-CDMA system using MRC and its comparison with AWGN channel. The performance for AWGN channel is obtained without considering the effect of fading on each subcarrier. The number of users used for comparison is 8 . It can be visualized from the plot that with increase in the fading parameter $m$, the probability of receiving the signal with less error increases, thereby increasing the system performance.

The simulated performance of MC CC-CDMA generated using SCC under frequency-selective Nakagami-m fading channel with different combining techniques is shown in Fig. 4. The comparison is with fading parameter $m=2$. The number of users used for comparison is 16. Here, the simulated performance of different combining schemes such as MRC, ORC, and MMSEC is compared using (11), (12), and (13). It can be observed that with more number of active users (interference limited system), the performance of ORC is better than that of MRC, but MMSEC offers diversity gain with knowledge of number of active users and noise power. Further, it can be seen that at Low SNR, MMSEC offers SNR gain of about $6 \mathrm{~dB}$ compared to ORC for BER of

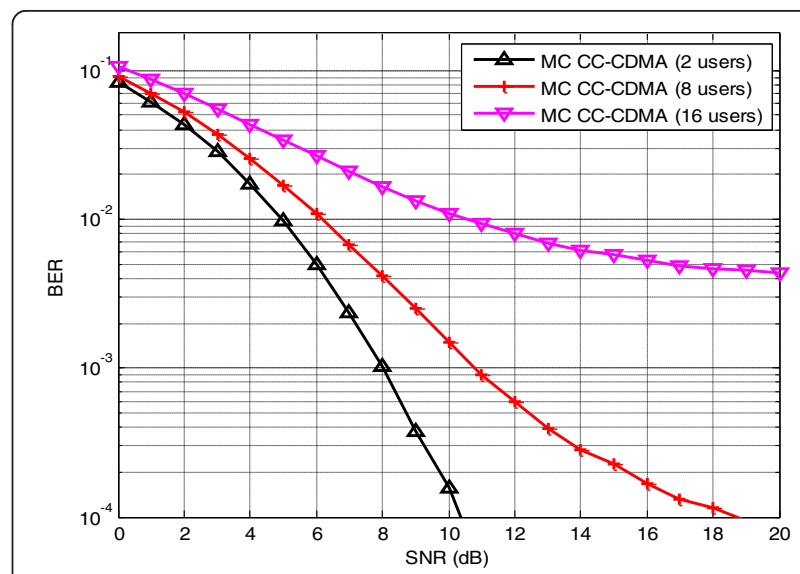

Fig. 2 BER performance comparison of MC CC-CDMA system using MRC under frequency-selective Nakagami-m fading channel with variations in number of users 


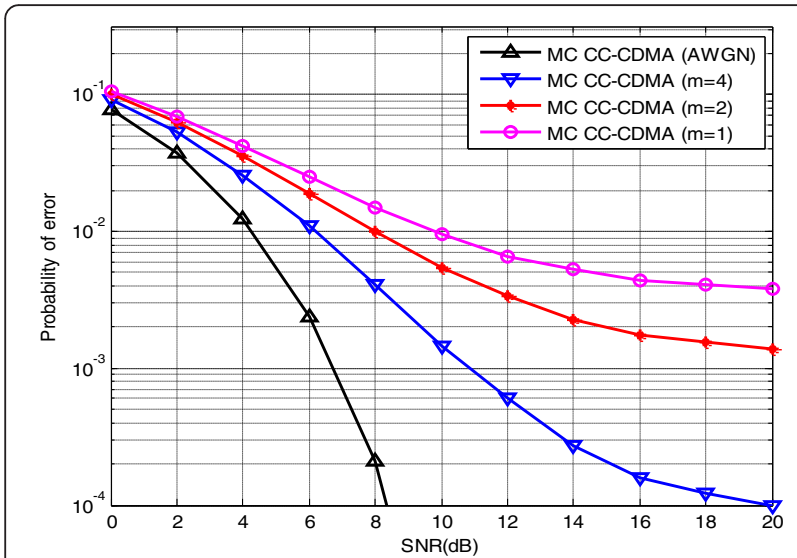

Fig. 3 BER performance comparison of MC CC-CDMA system with AWGN and MRC under frequency-selective Nakagami-m fading channel for different values of fading parameter $\mathrm{m}$

$10^{-2}$ and at high SNR, the SNR gain is twofold compared to ORC for BER of $10^{-3}$, respectively.

\subsection{B. BER performance comparison of MC CC-CDMA with PIC}

In this section, we discuss the error rate performance of MC CC-CDMA using different combining schemes with PIC to show the superiority of PIC in reducing the effect of MAI in frequency-selective Nakagami-m fading channel. The simulated BER performance of MC CC-CDMA is compared with analytical results to validate the theoretical results obtained. Further, we compare the performance of single carrier (SC) and multicarrier CDMA employing Walsh codes with multicarrier CC-CDMA to show the significance of complementary codes in obtaining larger diversity gain with PIC compared to noninterference cancellation techniques.

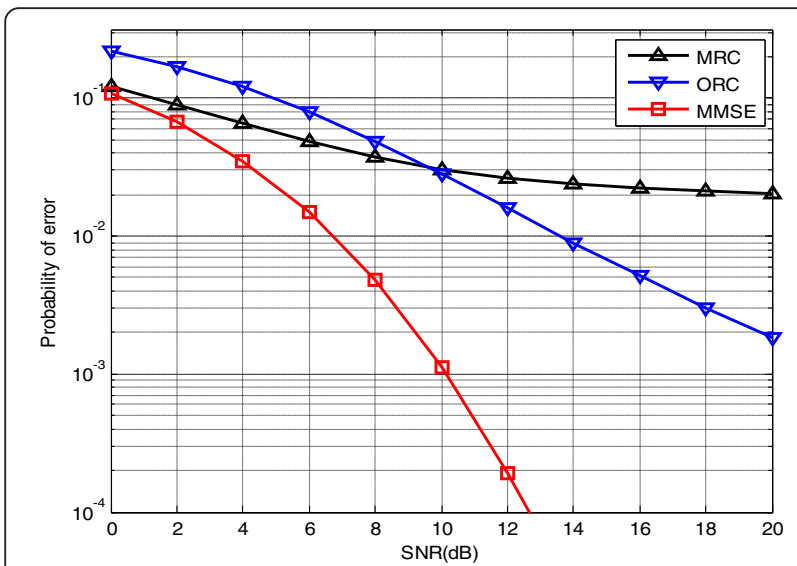

Fig. 4 Performance comparison of MC CC-CDMA using different combining schemes in frequency-selective Nakagami-m fading channel

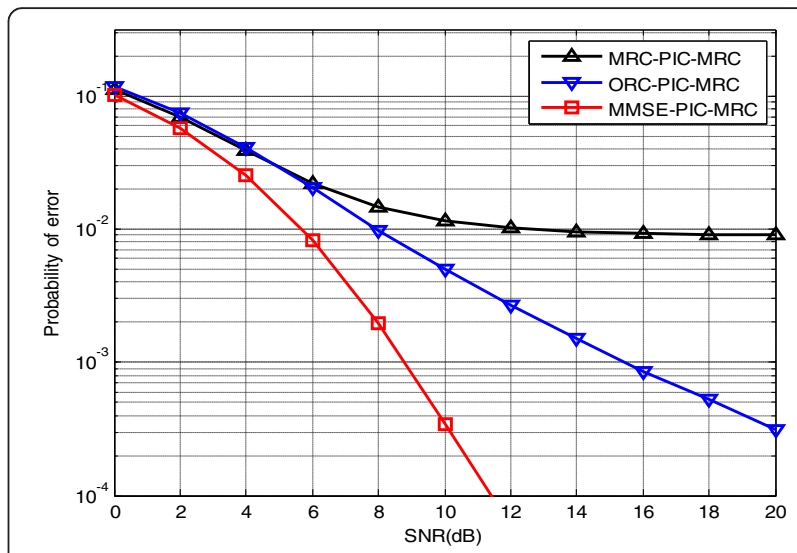

Fig. 5 Performance comparison of MC CC-CDMA using different interference cancellation schemes in frequency-selective Nakagami-m fading channel

Figure 5 shows the performance improvement achieved by using PIC with suitable combining schemes under frequency-selective fading channels using identical simulation parameters as in Fig. 4. The per-carrier parallel interference cancellation (PC-PIC) is performed in two stages. In the first stage, the initial data estimates of all interfering users are obtained using MRC, ORC, and MMSEC and PC-PIC is obtained using (15) resulting in MRC-PIC, ORC-PIC, and MMSEC-PIC. In the second stage, the interference-cancelled signal is dispread and combined using MRC to provide the desired users data. Here, the interference-cancelled signal using different interference estimation schemes is finally combined using MRC to obtain MRC-PIC-MRC, ORC-PIC-MRC, and MMSE-PICMRC. For instance, the notation MMSE-PIC-MRC refers to a PIC receiver using MMSE technique at the first stage and MRC at the second stage. It can be observed from Fig. 5 that the performance of PIC has a higher slope as compared to the system without interference cancellation (Fig. 4). This is mainly due to the reduction in MAI due to PIC. Further, it can be observed that at SNR of $10 \mathrm{~dB}$, the MC CC-CDMA with MRC-PIC-MRC, ORC-PIC-MRC, and MMSE-PIC-MRC provides BER of 0.0113, 0.0050, and 0.0003 , respectively, which is much lower than the error rate of MC CC-CDMA without PIC.

The analytical versus simulated performance comparison of MC CC-CDMA using PIC-based MRC under frequency-selective Nakagami-m fading channel is shown in Fig. 6. The simulated performance is obtained using OCC with set size, $N=16$ and $L c=4$ with PG of 64 for different parallel interference cancellation techniques such as MRC-PIC-MRC and MMSE-PIC-MRC. It can be observed that the simulated performance of MMSE-PIC-MRC agrees very well with the analytical result in (23) for the case of $m=6$ and $N=16$. This improvement in performance 


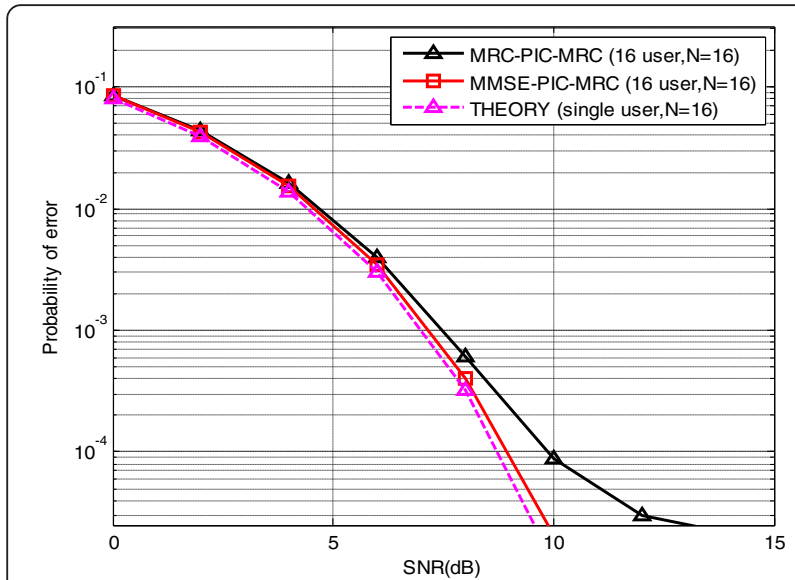

Fig. 6 Performance comparison of analytical and simulated BER for MC CC-CDMA using different interference cancellation techniques under frequency-selective Nakagami-m fading channels

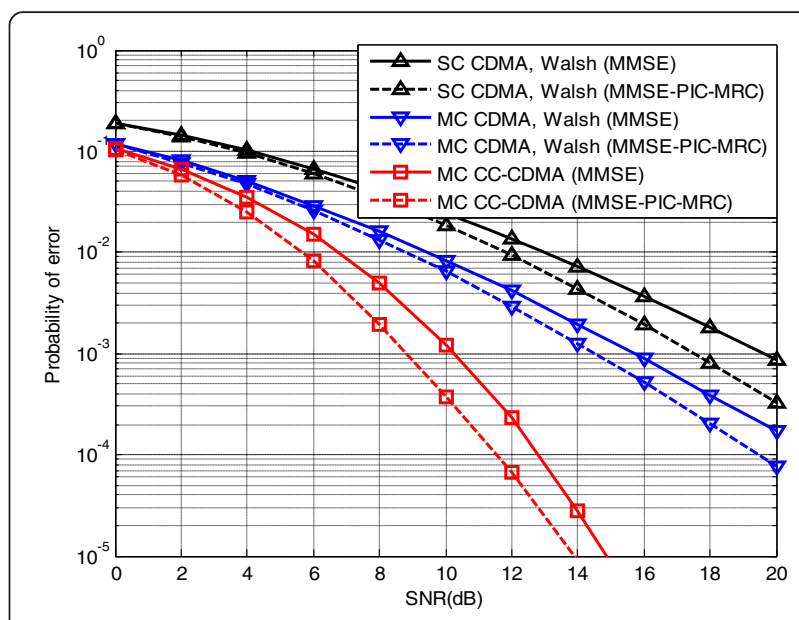

Fig. 8 BER performance comparison among SC-CDMA, MC-CDMA, and MC CC-CDMA with and without PIC under frequency-selective Nakagami-m fading channels is mainly due to the reduction in MAI due to MMSE-based PIC. Further, it can be visualized that for BER of $10^{-4}$, MMSE-PIC-MRC offers SNR gain of $3 \mathrm{~dB}$ compared to MRC-PIC-MRC, validating the superiority of MMSE-PIC-MRC in reducing the effect of MAI.

Figures 7 and 8 show the performance comparison of CDMA system using complementary codes with Walsh-coded CDMA to show the superiority of complementary codes in suppressing MAI in frequencyselective Nakagami-m fading channel. The PG of CC is 64 with element code length $L c=4$ and flock size $N=16$ with $m=2$. The PG of Walsh code is assumed to be 64 , and the number of users used is 16 for fair comparison. It can be observed from Fig. 7 that at BER of $10^{-3}$, with decrease in fading parameter $m$, there is not much degradation in performance of

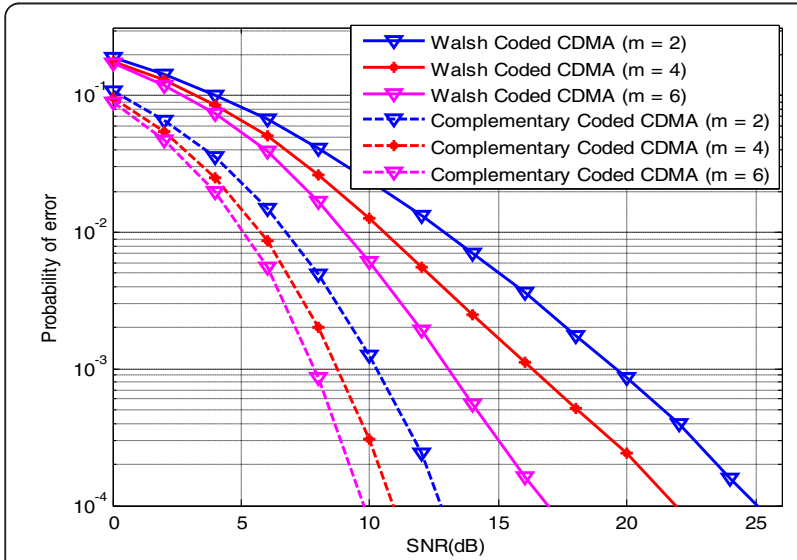

Fig. 7 BER performance comparison of CDMA systems using CC and Walsh code in frequency-selective fading channels with variations in fading parameter $m$ complementary-coded CDMA compared to Walsh-coded CDMA. At SNR of $10 \mathrm{~dB}$ and with $m=2$, it can be seen that complementary-coded CDMA employing MMSEPIC-MRC offers $10 \mathrm{~dB}$ improvement in performance gain compared to Walsh-coded CDMA providing controlled BER with reduced MAI.

In Fig. 8, we compare the BER performance of MC CC-CDMA with single carrier (SC) and multicarrier CDMA (MC-CDMA) employing Walsh code using MMSE combining and PIC in a frequency-selective Nakagami-m fading channel. The number of users considered in all the three systems is 16 and fading parameter $m$ is 2 . The number of subcarriers used for both MC-CDMA and MC CC-CDMA systems are 64 . It can be examined that the BER performance of $\mathrm{MC}$ CC-CDMA system using MMSE-PIC-MRC as a final combining scheme is more robust in frequencyselective Nakagami-m fading channels compared to other traditional SC and MC-CDMA systems using Walsh codes.

From Fig. 8, we can observe that the combining algorithms play a critical role in the performance of MC CCCDMA system. In addition, the use of PIC with different combining schemes in MC-CC-CDMA results in performance improvement at the cost of increased computational complexity compared to non-interference cancellation schemes. In MC-CDMA system employing Walsh codes and complementary codes, the per-carrier computational complexity of hard decision PIC detector as a function of users $K$ is $O(K(K-1))$, where $O($.) denotes the order of complexity [21]. Although the complexity incurred by both the systems are in the same order, MC CC-CDMA offers additional $8 \mathrm{~dB}$ gain at BER of $10^{-4}$ compared to MC CDMA using Walsh codes. This 
performance improvement is achieved due to additional diversity gain, which makes MC CC-CDMA suitable for system requiring higher data rate and capacity.

\section{Conclusions}

In this paper, we analyzed the performance of $\mathrm{MC}$ CC-CDMA system with different combining schemes for downlink transmissions under frequency-selective Nakagami-m fading channels. Performance measures in terms of BER versus SNR are plotted for different combining schemes to show the superiority of complementary codes in frequency-selective channels under different channel conditions. It was shown that in the presence of MAI, only MMSE-PIC with MRC can be more tolerant to interference with much higher gain than MRC-based PIC. Furthermore, the simulation results have revealed that an MC-CDMA system using $C C$ offers a higher diversity gain than traditional MC-CDMA systems using Walsh codes.

\section{Competing interests}

The authors declare that they have no competing interests.

\section{Acknowledgements}

The authors would like to thank the anonymous reviewers for their valuable comments which greatly improved the quality of the paper.

\section{Author details}

'Department of Electronics and Communication Engineering, St. Xavier's Catholic College of Engineering, Chunkankadai 629003Tamilnadu, India. ${ }^{2}$ Department of Electronics and Communication Engineering, DMI

Engineering College, Aralvaimozhi 627105, Tamilnadu, India.

Received: 21 August 2015 Accepted: 18 February 2016

Published online: 01 March 2016

\section{References}

1. KS Gilhousen, IM Jacobs, R Padovani, A Viterbi, On the capacity of cellular CDMA systems. IEEE Trans. Commun. 40(2), 303-312 (1991)

2. S Moshavi, Multiuser detection for DS-CDMA communications. IEEE Commun. Mag. 34, 124-136 (1996)

3. EH Dian, B Jabbari, Spreading codes for direct sequence CDMA and wideband CDMA cellular networks. IEEE Commun. Mag. 36(9), 143-146 (1998)

4. HH Chen, JF Yeh, N Suehiro, A multicarrier CDMA architecture based on orthogonal complementary codes for new generations of wideband wireless communications. IEEE Commun. Mag. 39(10), 126-135 (2001)

5. ME Magana, T Rajatasereekul, Complete complementary orthogonal (CCO) code based CDMA using natural mapping QAM constellations. Wireless Pers. Commun. 38(4), 435-442 (2006)

6. L Lu, V Dubey, Performance of a complete complementary code based spread-time CDMA system in a fading channel. IEEE Trans. Vehicular Technol. 57(1), 250-259 (2008)

7. H Chenggao, N Suehiro, T Hashimoto, A systematic frame work for the construction of optimal complete complementary codes. IEEE Trans. Inf. Theory 57(9), 6033-6042 (2011)

8. HH Chen, SW Chu, N Kuroyanagi, AJH Vinck, An algebraic approach to generate a superset of perfect complementary codes for interference-free CDMA. Wireless Commun. Mobile Comput. 79(5), 605-622 (2007)

9. HH Chen, SW Chu, M Guiziani, On next generation CDMA technologies: the real approach for perfect orthogonal code generation. IEEE Trans. Vehicular Technol. 57(5), 2822-2833 (2008)

10. HH Chen, The next generation CDMA technologies. (John Wiley and sons, NJ, USA, 2007)
11. WH Meng, SY Sun, HH Chen, Multiuser interference cancellation in complementary coded CDMA with diversity gain. IEEE Wireless Commun. Lett. 2(3), 303-306 (2013)

12. SY Sun, WH Meng, HH Chen, Uplink pre-equalization for CC-CDMA systems under frequency selective fading. Proc. of IEEE ICC. 2013, 5317-5321 (2013)

13. S Hara, R Prasad, Overview of multicarrier CDMA. IEEE Commun. Mag. 39(12), 126-133 (1997)

14. P Li, W Hamouda, Performance of multiple-input and multiple output orthogonal frequency and code division multiplexing systems in fading channels. IET Commun. 5, 1-11 (2012)

15. S Kaiser, On the performance of different detection techniques for OFDM-CDMA in fading channels. Proc. Globecom 3, 2059-2063 (1995)

16. S Verdu, Multiuser detection (Cambridge University Press, UK., 1998)

17. BA Al-fuhaidi, HEA Hassan, MM Salah, SS Alagooz, Parallel interference cancellation with different linear equalization and Rake receiver for the downlink MC-CDMA systems. IET Commun. 6(15), 2351-2360 (2012)

18. VA Aalo, Performance of maximal-ratio diversity system in a correlated Nakagami-faded environment. IEEE Trans. Commun. 43(8), 2360-2369 (1995)

19. JG Proakis, Digital communications, 4th edn. (McGraw Hill Inc, NY, 2001)

20. IS Gradshteyn, IM Ryzhik, Table of integrals, series and products, 6th edn. (Academic, San Diego, 2000)

21. DR Brown, Multistage parallel interference cancellation: convergence behavior and improved performance through limit cycle mitigation. IEEE Trans. Signal Process. 53(1), 283-294 (2005)

\section{Submit your manuscript to a SpringerOpen ${ }^{\circ}$ journal and benefit from:}

- Convenient online submission

- Rigorous peer review

- Immediate publication on acceptance

- Open access: articles freely available online

- High visibility within the field

- Retaining the copyright to your article

Submit your next manuscript at $\boldsymbol{\nabla}$ springeropen.com 